\title{
Comparative Analysis between Laparoscopic and Open IPOM Ventral Hernioplasty
}

\author{
Edzevit Kadri ${ }^{*} \quad$ Katarina Panikj $^{2}$ Dragoslav Mladenovic ${ }^{3} \quad$ Gjorgji Jota $^{4} \quad$ Jasminka Nanceva $^{5}$ \\ Svetozar Antovic $^{4} \quad$ Evgenija Lazova $^{1} \quad$ Zoran Dimitrov $^{6}$ \\ 1.City General Hospital 8 of September, NN Bledski dogovor 1000 Skopje, Republic of North Macedonia \\ 2.Public General Hospital Kochani, 21 Milan Zechar, 2300 Kochani, Republic of North Macedonia \\ 3.PHI University Clinic for Surgical Diseases St. Naum Ohridski, Boulevard 11 October, 1000 Skopje, \\ Republic of North Macedonia \\ 4.University Clinic for Digestive Surgery, 17 Mother Teresa, 1000 Skopje, Republic of North Macedonia \\ 5.University Clinic for Traumatology, Orthopaedic Diseases, Anaesthesia, Reanimation, Intensive Care and \\ Emergency Centre, 17 Mother Teresa, 1000 Skopje, Republic of North Macedonia \\ 6.Public General Hospital Gevgelija, 38 Slobodan Mitrov, 1480 Gevgelija, Republic of North Macedonia
}

\begin{abstract}
Introduction: In everyday surgical practice, ventral hernia repair is one of the most commonly performed surgeries worldwide. Ventral hernioplasty can be performed either with open or laparoscopic approach and in this study both approaches use the IPOM technique of mesh placement. From the clinical experience so far, the laparoscopic approach is characterized by a lower rate of early postoperative complications, shorter hospital stay and a period of convalescence.

The aim of the study was to compare early postoperative complications, duration of surgery, hospital stay, recurrence in the first year and convalescence period between patients undergoing open and laparoscopic access with IPOM ventral hernioplasty.

Material and methods: The study was designed as a randomized, prospective, comparative study of 63 patients who met the inclusion criteria, operated by IPOM technique and divided into two groups: open access to 32 patients and laparoscopic access to 31 patients. Early postoperative complications, duration of surgery, hospital stay, recurrence in the first year, and convalescence were compared in both groups. Statistical processing and data analysis was performed in the statistical programme SPSS version 23.0. Values of $p<0.05$ were taken as statistically significant.

Results: Regarding the early postoperative complications, it was found that the occurrence of seroma is more common in the laparoscopic group $(\mathrm{p}=0.013)$, while in open hernioplasty the number of surgical site infections is significantly higher $(p=0.03)$. The hospital stay expressed in days is longer in open hernioplasty $(p=000001)$. Regarding the duration of the operation $(\mathrm{p}=0.8)$ and the period of convalescence $(\mathrm{p}=0.28)$, there is no statistically significant difference between the two groups.

Discussion: The results shown by our experience shows that laparoscopic ventral IPOM hernia repair is characterized by lower rates of SSI, a shorter hospital stay, but without statistical significant benefit in terms of a period of convalescence, duration of surgery and recidive rate in the first year. The choice of treatment and access should be based according to individual patient characteristics. A laparoscopic approach, due to its minimal invasiveness, should be more common in ventral hernioplasty.
\end{abstract}

Keywords: Laparoscopic IPOM technique, open IPOM technique, ventral hernia, postoperative complications, SSI.

DOI: $10.7176 / \mathrm{JHMN} / 82-02$

Publication date: November $30^{\text {th }} 2020$

\section{Introduction}

Ventral hernia is a protrusion of intra-abdominal or preperitoneal contents through a defect in the fascia of the abdominal wall [1,2]. These defects can be congenital and acquired and since ventral hernias are a heterogeneous group, according to the European Society of Hernias (ENS) they are divided into primary - umbilical, epigastric, Spiegel and lumbar and incisional hernias [3]. Worldwide, ventral hernioplasty is one of the most commonly performed surgeries [4], but despite the high rate of these surgeries, there are still disagreements and controversies as to which is the ideal approach and method of hernioplasty. The laparoscopic approach of surgical treatment is generally associated with a lower rate of postoperative complications associated with the surgical site, a shorter hospital stay shortens the time to return to daily activities and increases patient satisfaction with the cosmetic effect. In1993, Le Blanc and Booth first described laparoscopic ventral hernioplasty. Regarding the treatment of ventral hernias, the advantages and disadvantages of the laparoscopic approach over the open one are still being analysed, although it is generally considered that the laparoscopic approach in ventral hernioplasty reduces the surgical complications.

Our study compares the advantages and disadvantages of open and laparoscopic approach to ventral 
hernioplasty, with mesh placement, in patients diagnosed and operated in one institution - City General Hospital " $8^{\text {th }}$ of September ", Skopje. Despite the different ways of setting up the mesh in the open method, but also in order to avoid the influence of the way of mesh placement as an impact factor on the results, in both approaches the IPOM technique was used (intraperitoneal " onlay " placement of the mesh).

\section{Objectives of the Study}

The aim of this comparative study is to compare whether there is a statistically significant difference in the incidence of early postoperative complications (in the first 30 days), by comparing the incidence of seroma, hematoma and surgical site infection, postoperative ileus, to compare the duration of operative surgery, hospital stay, recurrence in the first year and period of return to daily activities, between patients operated on with open and laparoscopic approach with IPOM ventral hernioplasty.

\section{Material and Methods}

\subsection{Study design}

The study is designed as a controlled, randomized, prospective, clinical, comparative study with predetermined protocol, which was conducted in PHI "CGH $8^{\text {th }}$ of September", Skopje, Republic of North Macedonia, in the period from March 2018 to March 2020. The study included 63 patients diagnosed with a ventral hernia (primary or incisional), operatively treated in the Digestive Surgery ward, who met the inclusion criteria of this study: age over 18 years, uncomplicated hernias, ASA classification I-III , defect size of $3-10 \mathrm{~cm}, \mathrm{BMI} \leq 40$ and signed informed consent. Patients were divided into two groups using computerized randomization on the day of preoperative examination and surgery scheduling and each patient was informed about the IPOM technique, hernioplasty approach and the differences between the open and the laparoscopic approach. The first group consisted of 32 patients with open IPOM technique, and the second group consisted of 31 patients, operated with laparoscopic IPOM technique. During the treatment of the patients, the treatment modalities included in the Protocols of EuraHS - European Register of Abdominal Wall Hernias, as well as the protocols for conducting safe surgery according to the recommendations of the Ministry of Health of RNM.

Patients were evaluated and compared with their demographic characteristics (gender, age, BMI), duration of surgery, early postoperative complications (seroma, hematoma, surgical site infections), postoperative ileus, length of hospital stay, period of convalescence and relapse in the first year.

\subsection{Surgical procedure}

All patients were operated under general anaesthesia and in all patients polypropylene composite mesh - Parietene with absorbent synthetic film (of various sizes) was used. In both groups, the mesh is placed intraperitoneally IPOM technique (intraperitoneal on-lay mesh), fixed with non-absorbent transfascial sutures (Prolene 0) and additionally with absorbable tackers in the laparoscopic group.

The open access surgical technique includes: incision at the site of the previous scar or hernia defect, adhesiolysis, measurement of the defect and plan for mesh placement, intraperitoneal placement of the mesh and fixation with transfacial sutures (on average 8 sutures) with overlap of 3-5 cm depending on the size of the defect, closing the defect (if possible), drainage in certain cases. $[9,10]$

The surgical technique for laparoscopic approach includes: pneumoperitoneum - by open method or with a Veress needle, placing working troacar as far away from the defect as possible, adhesiolysis, measuring the defect and placing the mesh intraperitoneally and fixation of the mesh with the transfascial sutures and absorbable tackers ("Double-Crown") at a distance of about $1.5-2.5 \mathrm{~cm} .[11,12]$

\section{Statistical analysis}

Statistical processing and data analysis was performed in the statistical programme SPSS for Windows 23.0. For testing of normality in the distribution of the data the Kolmogorov - Smirnov and Shapiro Wilk's test was used. Quantitative scores were represented by a standard deviation arithmetic mean and the groups were compared by the Student t-test and Mann-Whitnney test. The qualitative marks are shown in absolute and relative numbers and analysed by the Pearson Chi-square test. Values of $p<0.05$ were taken as statistically significant .

\section{Results}

Regarding the socio-demographic and clinical characteristics of the respondents, both groups of patients were homogeneous in terms of gender and age $(\mathrm{p}=0.38, \mathrm{p}=0.73)$, consistently ( Table 1). There was no gender difference between the two groups, although female patients were the majority of respondents in both groups $58.1 \%$ vs $68.75 \%$. The mean age was similar to patients with laparoscopic and open intervention $(53.64 \pm 13.7$ vs $54.65 \pm 9.5)$. Body mass index (BMI) was insignificantly presented with different values between the patients ( $\mathrm{p}$ $=0.95$ ) and was $2905 \pm 4.2 \mathrm{~kg} / \mathrm{m}^{2}$ in group that was undergoing laparoscopic hernia repair, and $28.99 \pm 3.4 \mathrm{~kg} / \mathrm{m}$

2 in the group that underwent open approach hernioplasty. 
Primary ventral hernia was insignificantly often diagnosed in patients undergoing laparoscopic hernia repair $-41.9 \%$ vs $28.1 \%$.

The study most often included patients with ASA score $2-64.5 \%$, and $53.1 \%$, respectively.

The size of the hernia defect did not differ significantly between patients with laparoscopic and open hernioplasty $(\mathrm{p}=0.09)$. The mean size of the hernia defect in the laparoscopic and open technique was 32 and $35 \mathrm{~cm}^{2}$, respectively.

Table 1. Description of the analysed groups of respondents

\begin{tabular}{|c|c|c|}
\hline Variable & laparoscopic & Open \\
\hline Age, years, $($ mean \pm SD) & $53.64 \pm 13.7$ & $54.65 \pm 9.5$ \\
\hline \multicolumn{3}{|l|}{ Gender $(\mathrm{n}(\%))$} \\
\hline Men & $13(41.94)$ & $10(31.25)$ \\
\hline Women & $18(58.06)$ & $22(68.75)$ \\
\hline BMI $($ mean \pm SD) & $29.05 \pm 4.2$ & $28.99 \pm 3.4$ \\
\hline \multicolumn{3}{|l|}{ hernia type (n (\%)) } \\
\hline Primary & $13(41.94)$ & $9(28.13)$ \\
\hline Incisional & $18(58.06)$ & $23(71.88)$ \\
\hline size of hernia defect ( mean \pm SD ) & $33.67 \pm 11.9$ & $35.55 \pm 19.4$ \\
\hline \multicolumn{3}{|l|}{ ASA classification $(\mathrm{n}(\%))$} \\
\hline 1 & $5(16.13)$ & $6(18.75)$ \\
\hline 2 & $20(64.52)$ & $17(53.13)$ \\
\hline 3 & $6(19.35)$ & $9(28.13)$ \\
\hline operation time ( mean $\pm \mathrm{SD}$ ) & $70.64 \pm 20.8$ & $75.75 \pm 29.1$ \\
\hline infections $(\mathrm{n}(\%))$ & $1(3.23)$ & $7(21.88)$ \\
\hline seroma $(n(\%))$ & $11(35.48)$ & $3(9.38)$ \\
\hline hematoma $(\mathrm{n}(\%))$ & $4(12.9)$ & $1(3.13)$ \\
\hline postoperative ileus (n (\%)) & $1(3.23)$ & $1(3.13)$ \\
\hline hospital days $($ mean \pm SD) & $3.06 \pm 0.9$ & $6.0 \pm 3.2$ \\
\hline recurrence in the first year $(\mathrm{n}(\%))$ & 0 & $2(6.25)$ \\
\hline Convalescence period (mean \pm SD) & $15.84 \pm 3.1$ & $16.78 \pm 3.7$ \\
\hline
\end{tabular}

$\chi^{2}$ (Chi-square test); $\mathrm{t}$ (Student t-test); Z (Mann-Whitney test)

The type of hernia repair does not significantly influence the duration of the intervention $(p=0.8)$. The operations lasted in average $70.64 \pm 20.8$ minutes in the group operated with laparoscopic approach, and $75.75 \pm$ 29.1 minutes in the group operated with open approach hernioplasty.

Regarding the incidence of events in the area of the operative wound (SSO - Surgical Site Occurrence ) in the first 30 days postoperatively, the results present: infection of the operative wound in one patient laparoscopically treated and in $21.9 \%$ (7) patients treated with open access ; seroma in 35.5\% (11) patients with laparoscopic and $9.4 \%$ (3) patients with open hernioplasty and hematoma in $12.9 \%$ (4) patients with laparoscopic hernioplasty and one patient with open hernioplasty.

Statistically significant are confirmed to be the frequent association of infection of the surgical site in open access hernia repair $(p=0.03)$ and the frequent association of seroma with the laparoscopic approach, while more common finding of hematoma in patients with laparoscopic versus open hernia repair was not statistically significant $(\mathrm{p}=0.196)$. Two patients had postoperative ileus, one in each group. The length of hospital stay of patients with a ventral hernia significantly depended on the type of hernioplasty $(p=0.000001)$. Patients who underwent laparoscopic method had significantly shorter hospitalization (mean $=3.06 \pm 0.9$ vs $6.0 \pm 3.2$ days, median $=3$ vs 5 days).

Recurrence of ventral hernia was reported in 2 patients in the group with open access hernioplasty.

The length of convalescence did not depend significantly on the surgical approach to hernioplasty $(p=0.28)$. Patients with laparoscopic approach had a slightly shorter postoperative recovery time than patients with classical approach $($ mean $=15.84 \pm 3.1$ vs $16.78 \pm 3.7 ;$ median $=15$ vs 16$)$.

\section{Discussion}

Ventral hernia is a common multifactorial disease that is treated surgically to prevent complications, such as pain, discomfort, intestinal obstruction, incarceration, and strangulation. When planning surgical treatment of a ventral hernia, primary or incisional, it is especially important to know well the pathogenesis of the hernia, the anatomy and physiology of the abdominal wall, the process of surgical wound healing including complications and to know well the surgical technique, which will be applied during the operative treatment.

Today there are numerous studies that emphasize the advantages of the laparoscopic approach over the open one, such as lower rate of early postoperative complications (surgical site infections, seroma, hematoma), better cosmetic effect, shorter hospital stay $[10,11]$. The German surgeon Ferdinand Kockerling in 2020 published a 
large comparative study of the open IPOM technique versus the sublay technique where it does not prove significant differences in terms of SSO, while the chronic pain is greater in the IPOM technique [12]. In another multicenter study, open IPOM technique in treatment of primary ventral hernia shows excellent results in terms of recurrence, pain, quality of life [13]. However, the same technique in laparoscopic hernioplasty has proven to be superior to open [14].

This study was designed as a controlled, randomized, prospective, clinical, comparative study. It included 63 patients divided into two comparative groups, previously randomized by a computer programme. Both groups of patients were homogeneous in terms of demographic characteristics- gender and age, BMI. Most patients in the preoperative anaesthesia assessment belonged to the ASA 2 classification. There were no statistically significant differences between the type of hernia and the size of the hernia defect in both groups.

To avoid the bias that would occur during the operation, all patients were operated by the same surgeon or with the assistance (active participation) of the same surgeon. This eliminates the possibility of greater variations in the technique and duration of the surgery that would occur when working with different surgeons that would depend on their training and individual abilities.

Also, in order to eliminate the influence of the mesh position on the results, in both groups (laparoscopic and open) the mesh was placed intraperitoneally (IPOM method).

According to the presented results, this study showed that in patients treated with laparoscopic approach with IPOM method, the occurrence of seroma is statistically significant, which according to previous judges occurs more often after laparoscopic approach [15]. On the other hand, the open group patients had a significantly higher rate of surgical site infection (SSI). Numerous studies have shown that surgical site infections are associated with a higher recurrence rate of hernias, longer hospital stays, and a higher incidence of re-hospitalization [16]. Infection of the surgical site after ventral hernioplasty, open or laparoscopic, is a highly morbid complication associated with additional financial burden, relapse, and mesh infection with its complications [17,18]. Patients operated with laparoscopic approach have a shorter hospital stay. Regarding the duration of the operation and the period of convalescence, no statistically significant differences were observed between the two groups. Recurrence was noted in 2 patients in the open group.

Finally, the choice of treatment and access for ventral hernia repair should be based according to the individual characteristics of the patient and be based on evidence and accepted consensus and foremost promote minimally invasive surgery as treatment with the most benefits for the patient's health.

\section{References}

1. Townsend RC, Beauchamp BD, Mattox MEK. Clinical surgery of hernia. Sabiston Textbook of Surgery, 19th Edition, Volume II, Elsevier. 2016: 1128.

2. Brunicardi CF, Andersen DK, Billiar TK, et al. Schwartz's Principles of Surgery. 9. New York: McGraw-Hill Companies, Inc.; 2010. p. 1272.

3. Muysoms FE, Miserez M, Berrevoet F, Campanelli G, Champault GG, Chelala E, Dietz UA, Eker HH, El Nakadi I, Hauters P, Hidalgo Pascual M, Hoeferlin A, Klinge U, Montgomery A, Simmermacher RK, Simons MP, Smietański M, Sommeling C, Tollens T, Vierendeels T, Kingsnorth A. Hernia. 2009 Aug; 13 (4): $407-$ 14.

4. Laparoscopic versus open ventral hernia repair. Jin J, Rosen MJ. Surg Clin North Am. 2008 Oct; 88 (5): 1083 100, viii.

5. Laparoscopic repair of incisional abdominal hernias using expanded polytetrafluoroethylene: preliminary findings. LeBlanc KA, Booth WV; Surg Laparosc Endosc. 1993 Feb; 3 (1): 39-41.

6. Jones, Daniel B, Master Techniques in Surgery: Hernia, Lippincott Williams \& Wilkins (LWW), 1st Ed, 2012 , p.245-385.

7. Köckerling F and Lammers B (2018): Open Intraperitoneal Onlay Mesh (IPOM) Technique for Incisional Hernia Repair. Front. Surg. 5: 66.

8. Karl A. LeBlanc, MD . Current Considerations in Laparoscopic Incisional and Ventral Herniorrhaphy, JSLS. 2000 Apr-Jun; 4 (2): 131-139

9. Mohamed WBA et al . Laparoscopic versus open incisional hernia repair: comparative nonrandomized study , IntSurg J. 2017 Jul; 4 (7): 2216-2220

10. Forbes SS, Eskicioglu C, McLeod RS et al. Meta-analysis of randomized controlled triales comparing open and laparoscopic ventral and incisional hernia repair with mesh. Br J Surg 2009; 96: 851-8.

11. McGreevy JM, Goodney PP, Birkmeyer CM, Finlayson SRG, Laycock WS, Birkmeyer JD. A prospective study comparing the complication rates between laparoscopic and open ventral hernia repairs. Surg. Endosc. 2003; 17: 1778-1780.

12. Köckerling F, Lammers B, Weyhe D, et al. What is the outcome of the open IPOM versus sublay technique in the treatment of larger incisional hernias?: A propensity score-matched comparison of 9091 patients from the Hernia med Registry ; Hernia . 2020; 10.1007 / s10029-020-02143-4. 
13. Berrevoet F, Doerhoff C, Muysoms F, et al. Open ventral hernia repair with a composite ventral patch - final results of a multicentre prospective study. BMC Surg . 2019; 19 (1): 93.

14. Lavanchy JL, Buff SE, Kohler A, Candinas D, Beldi G. Long-term results of laparoscopic versus open intraperitoneal onlay mesh incisional hernia repair: a propensity score-matched analysis. Surg Endosc. 2019; 33 (1): 225-233.

15. https:/www.sages.org/publications/guidelines/guidelines-for-laparoscopic-ventral-hernia-repair/

16. Berger RL, Li LT , Hicks SC, Davila JA, Kao LS, Liang MK . Development and validation of a riskstratification score for surgical site occurrence and surgical site infection after open ventral hernia repair. $\mathrm{J}$ Am Coll Surg, 2013 Dec; 217 (6): 974-82.

17. Sara A Hennessy, Tjasa Hranjec, MS, Peter T Hallowell, M, Bruce D Schirmer . Surgical site infections following ventral hernia repair: Does operative approach matter? https://www.sages.org/meetings/annualmeeting/abstracts-archive/ surgical-site-infections-following-ventral-hernia-repair-does-operative-approachmatter /

18. Murray BW, Cipher DJ, Pham T, Anthony T. The impact of surgical site infection on the development of incisional hernia and small bowel obstruction in colorectal surgery. Am J Surg. 2011; 202 (5): 558-560. 